\title{
Planar open books with four binding components
}

\author{
YANKI LEKILI
}

\begin{abstract}
We study an explicit construction of planar open books with four binding components on any three-manifold which is given by integral surgery on three component pure braid closures. This construction is general, indeed any planar open book with four binding components is given this way. Using this construction and results on exceptional surgeries on hyperbolic links, we show that any contact structure of $S^{3}$ supports a planar open book with four binding components, determining the minimal number of binding components needed for planar open books supporting these contact structures. In addition, we study a class of monodromies of a planar open book with four binding components in detail. We characterize all the symplectically fillable contact structures in this class and we determine when the Ozsváth-Szabó contact invariant vanishes. As an application, we give an example of a right-veering diffeomorphism on the four-holed sphere which is not destabilizable and yet supports an overtwisted contact structure. This provides a counterexample to a conjecture of Honda, Kazez and Matić from [16].
\end{abstract}

$57 \mathrm{R} 17$

\section{Introduction}

Let $Y$ be a closed oriented 3-manifold and $\xi$ be a contact structure on $Y$. Recall that an open book is a fibration $\pi: Y-B \rightarrow S^{1}$ where $B$ is an oriented link in $Y$ such that the fibres of $\pi$ are Seifert surfaces for $B$. The contact structure $\xi$ is said to be supported by an open book $\pi$ if $\xi$ is the kernel of a one-form $\alpha$ such that $\alpha$ evaluates positively on the positively oriented tangent vectors of $B$ and $d \alpha$ restricts to a positive area form on each fibre of $\pi$. The fibres of $\pi$ are called pages of the open book. We will consider abstract open books $(S, \phi)$ where $S$ is a page of the open book, and $\phi \in \operatorname{Aut}(S, \partial S)$. It is easy to construct an open book as above, starting from the data $(S, \phi)$ (see Etnyre [7]).

It is well known that every contact structure $\xi$ is supported by an open book on $Y$ and all open book decompositions of $Y$ supporting $\xi$ are equivalent up to positive stabilizations and destabilizations (see Giroux [12]). In light of this theorem, to study contact structures, we will study abstract open books $(S, \phi)$ supporting them. We 
should note that in our case the right notion of equivalence provided by the Giroux's theorem is contact isomorphism (not contact isotopy [7]).

In [6], Etnyre proved that every overtwisted contact structure is supported by a planar open book. On the other hand, there are known obstructions for a tight contact structure to admit a supporting planar open book (see Etnyre [6], Ozsváth, Stipsicz and Szabó [20] and Wand [24]).

For a contact structure $(Y, \xi)$, in [8], Etnyre and Ozbagci defined invariants of $\xi$ by a measure of topological complexity of its supporting open books. We recall these here:

$$
\begin{aligned}
\operatorname{sn}(\xi) & =\min \left\{-\chi\left(\pi^{-1}(\theta)\right) \mid \pi: Y-B \rightarrow S^{1} \text { supports } \xi\right\} \\
\operatorname{sg}(\xi) & =\min \left\{g\left(\pi^{-1}(\theta)\right) \mid \pi: Y-B \rightarrow S^{1} \text { supports } \xi\right\} \\
\operatorname{bn}(\xi) & =\min \left\{|B| \mid \pi: Y-B \rightarrow S^{1} \text { supports } \xi \text { and } g\left(\pi^{-1}(\theta)\right)=\operatorname{sg}(\xi)\right\}
\end{aligned}
$$

where $\theta$ is any point in $S^{1}, g(\cdot)$ is the genus and $|\cdot|$ is the number of components.

These are called support norm, support genus and binding number in the order given above. In general, it is hard to compute these invariants for a given $\xi$. From the above definition, it is easy to see that $\operatorname{sn}(\xi) \leq 2 \operatorname{sg}(\xi)+\operatorname{bn}(\xi)-2$, however it is known that in general these invariants are independent of each other (see Etgü and Lekili [5] and Baldwin and Etnyre [4]).

In this article, we will determine all of these invariants for all the contact structures on $S^{3}$. Previously for any contact structure $\xi$ on $S^{3}$, Etnyre and Ozbagci showed that $\operatorname{sg}(\xi)=0, \operatorname{bn}(\xi) \leq 6$ and $\operatorname{sn}(\xi) \leq 4$. Recall that, there exists a unique tight contact structure on $S^{3}$ having $d_{3}=-1 / 2$. It is easy to show that this is supported by the open book $\left(D^{2}\right.$, id $)$, hence $\mathrm{sg}=0, \mathrm{bn}=1$ and $\mathrm{sn}=-1$ for the tight contact structure on $S^{3}$. The overtwisted contact structures on $S^{3}$ are classified by their $d_{3}$ invariants which takes values in $\mathbb{Z}+1 / 2$. We will write $\xi_{n}$ for the overtwisted contact structure on $S^{3}$ with $d_{3}=n$. Our first result determines the invariants of these:

Theorem 1.1 Let $\xi_{n}$ be the overtwisted contact structure on $S^{3}$ with $d_{3}\left(\xi_{n}\right)=n$, then $\operatorname{sg}\left(\xi_{n}\right)=0$ for all $n$ and

$$
\begin{array}{rlrl}
\operatorname{bn}\left(\xi_{1 / 2}\right) & =2 & \\
\operatorname{bn}\left(\xi_{-1 / 2}\right) & =\operatorname{bn}\left(\xi_{3 / 2}\right)=3 & \\
\operatorname{bn}\left(\xi_{n}\right) & =4 & & \text { for all } n \neq-1 / 2,1 / 2,3 / 2 \\
\operatorname{sn}\left(\xi_{1 / 2}\right) & =0 & & \\
\operatorname{sn}\left(\xi_{-1 / 2}\right) & =\operatorname{sn}\left(\xi_{3 / 2}\right)=1 & \\
\operatorname{sn}\left(\xi_{n}\right) & =2 & & \text { for all } n \neq-1 / 2,1 / 2,3 / 2 .
\end{array}
$$


Note that the results for $n=-1 / 2,1 / 2,3 / 2$ were calculated by Etnyre and Ozbagci [8] via an easy classification of planar open books with three or less boundary components, which we review here. Let $(Y, \xi)$ be the contact three-manifold supported by $(S, \phi)$. Below, we write $\left(Y, \xi_{\text {st }}\right)$ to denote the unique tight contact structure on $Y$ whenever $Y$ has a unique tight contact structure. These descriptions and more can be found in [8].

- If $S=D^{2}$, then $\phi=$ id and $(Y, \xi)=\left(S^{3}, \xi_{\text {st }}\right)$.

- For $S=S^{1} \times[0,1]$, let $a$ denote the simple closed curve generating $H_{1}(S)$. If $\phi=\tau_{a}^{p}$, then $(Y, \xi)=\left(L(p, p-1), \xi_{\mathrm{st}}\right)$ for $p>0,(Y, \xi)=\left(S^{1} \times S^{2}, \xi_{\mathrm{st}}\right)$ for $p=0$, and $(Y, \xi)=(L(-p, 1), \xi)$ for $p<0$, where $\xi$ is overtwisted with $e(\xi)=0$ and $d_{3}(\xi)=(3+p) / 4$. Note that $S^{3}$ appears exactly for $p= \pm 1$. For $p=1$, this is a stabilization of the standard open book of tight contact structure in $S^{3}$, and for $p=-1$, we get the overtwisted contact structure $\xi_{1 / 2}$.

- When $S$ has three boundary components, let $a, b, c$ denote boundary parallel simple closed curves. If $\phi=\tau_{a}^{p} \tau_{b}^{q} \tau_{c}^{r}$, then $Y$ is the Seifert fibered space with $e_{0}=\lfloor-1 / p\rfloor+\lfloor-1 / q\rfloor+\lfloor-1 / r\rfloor$ as shown in Figure 1 . We only note that it is easy to draw a contact surgery diagram of these contact structures [8]. The authors calculate exactly when $S^{3}$ has such an open book, it turns out all of these open books support either $\xi_{-1 / 2}$ or $\xi_{3 / 2}$.

To determine $\operatorname{bn}\left(\xi_{n}\right)$ for the remaining cases, we simply construct planar open books with four binding components supporting $\xi_{n}$ for the remaining cases. This determines $\operatorname{bn}\left(\xi_{n}\right)$. To calculate $\operatorname{sn}\left(\xi_{n}\right)$, we show that none of these contact structures can be supported by an open book with page a torus with one boundary component.
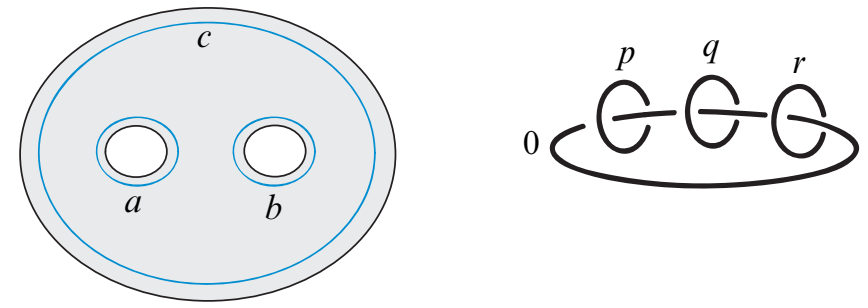

Figure 1: Open books with page a three-holed sphere

In [15], Honda, Kazez and Matić proves that a contact structure $\xi$ is tight if and only if all of the open book decompositions $(S, \phi)$ supporting $\xi$ have right-veering monodromy $\phi \in \operatorname{Aut}(S, \partial S)$. This result is useful in proving that $\xi$ is overtwisted by exhibiting a supporting open book with a monodromy which is not a right-veering diffeomorphism. On the other hand, when $S$ is a punctured torus, the same authors 
in [16] also prove that the supported contact structure is tight if and only if the given monodromy is right-veering. In general, however a right-veering diffeomorphism does not always correspond to a tight contact structure. In fact, any open book can be stabilized to a right-veering one. However, Honda, Kazez and Matić optimistically conjecture that if the monodromy is given by a right-veering diffeomorphism that does not admit a destabilization (in the sense of Giroux stabilization) then the supported contact structure is tight. Our next result gives a counterexample to this conjecture:

Theorem 1.2 There exists an open book $(S, \phi)$ on the Poincare homology sphere $\Sigma(2,3,5)$ where $S$ is a four-holed sphere and $\phi=\tau_{a}^{5} \tau_{b}^{2} \tau_{c} \tau_{d} \tau_{e}^{-2}$ which is right-veering and not destabilizable such that the supported contact structure is an overtwisted contact structure.

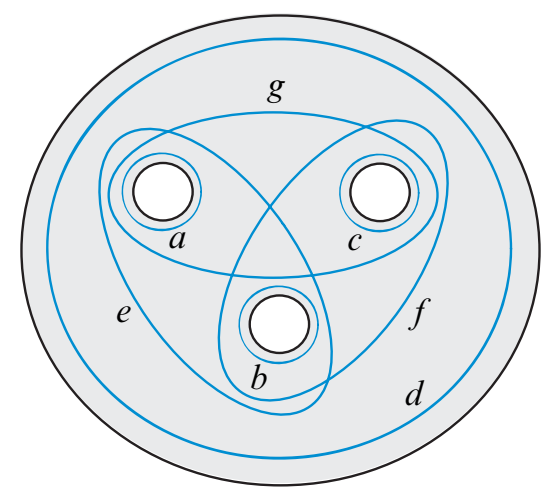

Figure 2: Generators of the mapping class group of four-holed sphere

Of independent interest, we also prove the following characterization concerning positive factorizations of a family of elements in the mapping class group of fourholed sphere. We denote by $c^{+}(\phi)$ the Ozsváth-Szabó contact invariant of the contact structure supported by $(S, \phi)$, where $S$ is the four-holed sphere.

Theorem 1.3 If $\phi=\tau_{a}^{\alpha} \tau_{b}^{\beta} \tau_{c}^{\gamma} \tau_{d}^{\delta} \tau_{e}^{\epsilon} \tau_{f}^{\eta}$, then $\phi$ admits a positive factorization if and only if $\min \{\alpha, \beta, \gamma, \delta\} \geq \max \{-\epsilon,-\eta, 0\}$. Furthermore, this latter condition is satisfied if and only if $c^{+}(\phi) \neq 0$.

Note that the results of Wendl [25] and Niederkrüger and Wendl [19] together with Theorem 1.3 imply that the contact structure supported by $\left(S, \tau_{a}^{\alpha} \tau_{b}^{\beta} \tau_{c}^{\gamma} \tau_{d}^{\delta} \tau_{e}^{\epsilon} \tau_{f}^{\eta}\right)$ admits a Stein filling (or equivalently a weak-symplectic filling) if and only if $\min \{\alpha, \beta, \gamma, \delta\} \geq$ $\max \{-\epsilon,-\eta, 0\}$. An interesting question left open is whether all nonfillable contact 
structures in the class of monodromies considered above are overtwisted. Note that one can easily show that some monodromies give overtwisted contact structures by showing that they are not right-veering, however Theorem 1.2 shows that right-veering restriction by itself is not enough to answer this question.

We pause here to declare our conventions for the rest of the paper. We denote by $\tau_{a}$ a right-handed Dehn twist about the curve $a$. We will adhere to braid notation for compositions: $\tau_{a} \tau_{b}$ means applying a right-handed Dehn twist about $a$ first and then a right-handed Dehn twist about $b$. We will also use the following conventions for braid groups: Our braids will be drawn from top to bottom with the strands numbered $1,2, \ldots, n$ from left to right. The convention for positive and negative half twist is as shown below.

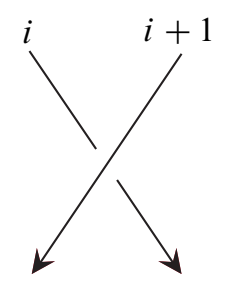

$\sigma_{i}$

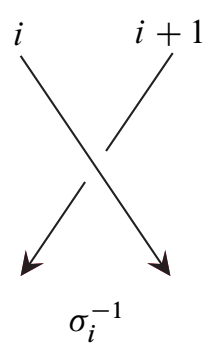

$\sigma_{i}^{-1}$

Figure 3: Braid group generators

Acknowledgements I would like to thank Andy Wand for helpful conversations, Tolga Etgü and John Etnyre for useful comments on a previous draft. I also acknowledge Max-Planck Institut für Mathematik for the support.

\section{A topological study of planar open books}

\subsection{Planar open books and Dehn surgery}

We first recall a classical proposition relating the mapping class group of a $n$-holed disk with Dehn surgery on pure braids (see for example Prasalov and Sossinsky [22] for more than presented here). Let $\phi$ be a diffeomorphism of the $n$-holed disk, the identity on the boundary. This diffeomorphism can be extended to a diffeomorphism $\widetilde{\phi}$ of the disk simply by extending $\phi$ by the identity. Since any diffeomorphism of the disk which is the identity on the boundary is isotopic to identity, there exists an isotopy $\widetilde{\phi}_{t}$ such that $\widetilde{\phi}_{0}=$ id and $\widetilde{\phi}_{1}=\widetilde{\phi}$. Let $x_{1}, \ldots, x_{n}$ be points in the disks that fill the holes, then we obtain a pure braid $\beta(\phi)$ by considering the union of $\operatorname{arcs}\left(\tilde{\phi}_{t}\left(x_{i}\right), t\right)$ in 
$D^{2} \times[0,1], t \in[0,1]$ (see Figure 4 for an example). This pure braid almost captures the whole $\phi$, except $\phi$ can have extra boundary twists around the holes. We summarize this in the proposition below. Let $D_{n}$ denote the $n$ holed disk, and $\operatorname{Map}\left(D_{n}, \partial D_{n}\right)$ be the mapping class group of diffeomorphisms which are identical on the boundary. Let $P_{n}$ be the pure braid group on $n$ strands.

Proposition 2.1 $\operatorname{Map}\left(D_{n}, \partial D_{n}\right)=P_{n} \times \mathbb{Z}^{n}$
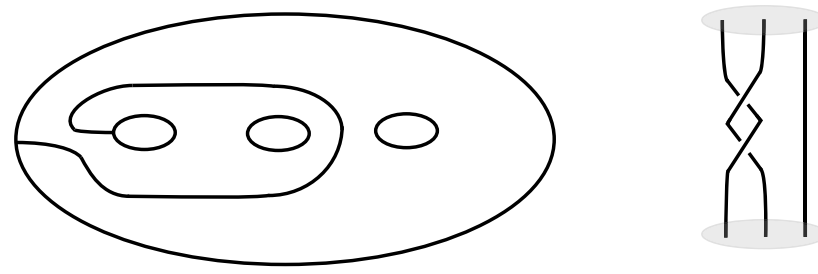

Figure 4: Pure braid associated with a mapping class

Note that $n$-holed disk is topologically the same as $(n+1)$-holed sphere, however the above isomorphism is meaningful only after choosing a boundary component of the $(n+1)$-holed sphere to be identified with the boundary of $D^{2}$ after filling in the other boundary components with disks. Nevertheless, such a choice can be made once and for all. By looking at Figure 2, we choose the boundary component parallel to the curve $d$ to correspond to the boundary of $D^{2}$, and the pure braid will be obtained by filling in the boundary components parallel to the curves $a, b$ and $c$, in addition we choose the ordering of the strands of the pure braid in this order. This is illustrated in Figure 4. On the left, we see an illustration of the right-handed Dehn twist about a curve encircling the first two holes, and on the right we see the corresponding pure braid.

This proposition gives us an alternative way to describe the underlying topological manifold supported by an open book $\left(D_{n}, \phi\right)$. Namely if $Y$ has an open book $\left(D_{n}, \phi\right)$, then $Y$ is obtained by Dehn surgery on the braid closure $\widehat{\beta}(\phi)$ of the braid $\beta(\phi)$ with surgery coefficients determined by the above isomorphism.

In this article, we study planar open books with four binding components. For the sake of explicitness, we give a more precise statement of the above discussion for this case.

Let $S=D_{3}$ denote a four-holed sphere, the mapping class group $\operatorname{Map}(S, \partial S)$ is not a free abelian group in contrast to the case three-holed sphere, in particular it has a subgroup isomorphic to $F_{2}$, the free group on two generators, generated by Dehn twists around $e$ and $f$ in Figure 2. In fact, it is a classical fact that $\operatorname{Map}(S, \partial S)$ can 
be seen as a direct product $\mathbb{Z}^{4} \times F_{2}$ (see Fadell and Neuwirth [9]). We can see this as follows: Because of Proposition 2.1, it suffices to see that $P_{3}$ is $\mathbb{Z} \times F_{2}$. Recall that $P_{3}$ is isomorphic to the fundamental group of the space of triples of distinct points on the plane (see Fox and Neuwirth [10]). Consider the forgetful map, from $P_{3} \rightarrow P_{2}$, given by forgetting about the middle strand. $P_{2}$ is $\mathbb{Z}$ and the kernel of this map is $\pi_{1}(\mathbb{C}-\{-1,1\}, 0)$, which is $F_{2}$. Thus we have a short exact sequence

$$
0 \rightarrow F_{2} \rightarrow P_{3} \rightarrow \mathbb{Z} \rightarrow 0
$$

where the kernel is generated by $\sigma_{1}^{2}$ and $\sigma_{2}^{2}$, and the image is generated by the central element $\left(\sigma_{2} \sigma_{1} \sigma_{2}\right)^{2}$ which corresponds to a full right-handed twist of the three-strands. Thus, any pure 3-braid is expressed uniquely as $\left(\sigma_{2} \sigma_{1} \sigma_{2}\right)^{2 \delta} \sigma_{1}^{2 \epsilon_{1}} \sigma_{2}^{2 \eta_{1}} \cdots \sigma_{1}^{2 \epsilon_{k}} \sigma_{2}^{2 \eta_{k}}$, where $\delta, \epsilon_{i}, \eta_{i}$ are integers.

Therefore, under the identification of Proposition 2.1 any mapping class $\phi \in \operatorname{Map}(S, \partial S)$ can be represented by

$$
\phi=\tau_{a}^{\alpha} \tau_{b}^{\beta} \tau_{c}^{\gamma} \tau_{d}^{\delta} \tau_{e}^{\epsilon_{1}} \tau_{f}^{\eta_{1}} \cdots \tau_{e}^{\epsilon_{k}} \tau_{f}^{\eta_{k}}
$$

and such a representation is unique.

Here, $\phi$ is identified with the pure braid $\beta(\phi)=\left(\sigma_{2} \sigma_{1} \sigma_{2}\right)^{2 \delta} \sigma_{1}^{2 \epsilon_{1}} \sigma_{2}^{2 \eta_{1}} \cdots \sigma_{1}^{2 \epsilon_{k}} \sigma_{2}^{2 \eta_{k}}$, and the integers $(\alpha, \beta, \gamma)$. For such open books, we have the following proposition as part of the general discussion above:

Proposition 2.2 Let $S$ be the four-holed sphere and $\phi=\tau_{a}^{\alpha} \tau_{b}^{\beta} \tau_{c}^{\gamma} \tau_{d}^{\delta} \tau_{e}^{\epsilon_{1}} \tau_{f}^{\eta_{1}} \cdots \tau_{e}^{\epsilon_{k}} \tau_{f}^{\eta_{k}}$. Let $\epsilon=\sum_{i=1}^{k} \epsilon_{i}$ and $\eta=\sum_{i=1}^{k} \eta_{i}$. Then the topological manifold $Y$ given by the open book $(S, \phi)$ can be obtained by Dehn surgery on the braid closure of the pure 3 -braid $\beta=\left(\sigma_{2} \sigma_{1} \sigma_{2}\right)^{2 \delta} \sigma_{1}^{2 \epsilon_{1}} \sigma_{2}^{2 \eta_{1}} \cdots \sigma_{1}^{2 \epsilon_{k}} \sigma_{2}^{2 \eta_{k}}$, with surgery coefficients $(\alpha+\delta+\epsilon$, $\beta+\delta+\epsilon+\eta, \gamma+\delta+\eta)$.

\subsection{Planar open books on the three-sphere}

We would like to construct planar open books with four boundary components on $S^{3}$. We will look for planar open books with simple monodromy having the form $\phi=$ $\tau_{a}^{\alpha} \tau_{b}^{\beta} \tau_{c}^{\gamma} \tau_{d}^{\delta} \tau_{e}^{\epsilon} \tau_{f}^{\eta}$. In light of Proposition 2.2, we would like to know when a surgery on a braid closure of a pure 3-braid of the form $\beta=\left(\sigma_{2} \sigma_{1} \sigma_{2}\right)^{2 \delta} \sigma_{1}^{2 \epsilon} \sigma_{2}^{2 \eta}$ yields $S^{3}$. Fortunately, this question is completely resolved by Armas-Sanabria and Eudave-Muñoz in [2] by depending on deep results on Dehn surgery on knots. In particular, the authors list several infinite families. Therefore, we can describe precisely when an open book $\left(S, \tau_{a}^{\alpha} \tau_{b}^{\beta} \tau_{c}^{\gamma} \tau_{d}^{\delta} \tau_{e}^{\epsilon} \tau_{f}^{\eta}\right)$ is an open book on $S^{3}$. 
From the list provided in [2] we pick a convenient family. By using Kirby calculus, we will verify independently that these indeed give $S^{3}$, and our next task is to calculate the $d_{3}$ invariants of the contact structures supported by the corresponding open books. The difficulty is that we would like to see that any value in $\mathbb{Z}+1 / 2$ can be achieved. We will apply several tricks to ensure this. Therefore, as a consequence of these calculations, we show that every contact structure on $S^{3}$ is supported by an open book with a planar page with at most four binding components.

Proof of Theorem 1.1 We will start with the braid $\beta=\left(\sigma_{2} \sigma_{1} \sigma_{2}\right)^{2} \sigma_{1}^{-4} \sigma_{2}^{-4}$. Figure 5 is a picture of the closure of this braid, also known as the chain link. The hyperbolic structure on its complement was first constructed by Thurston in his notes [23], and this manifold has been called as the "magic manifold" by Gordon and Wu $[13 ; 14]$ as Dehn fillings of this complement recover many of the known hyperbolic manifolds and account for most of the interesting nonhyperbolic fillings of cusped hyperbolic manifolds (see Martelli and Petronio [18] for a classification of all exceptional surgeries on this link). It is the 3-cusped hyperbolic manifold with smallest known volume and complexity by Adams and Sherman [1].

It is easy to see by blowing down twice that $(-1,-2,-4)$ surgery on this link yields $S^{3}$ (see below for the more general case).

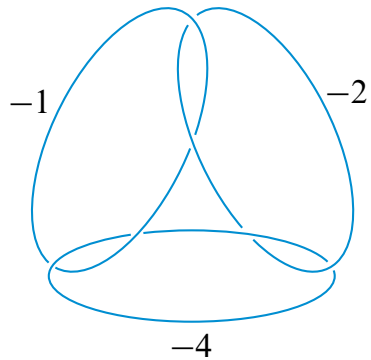

Figure 5: Surgery on the chain link

Therefore, by our Proposition 2.2, it follows that the open book with page $S$, a fourholed sphere, and $\phi=\tau_{a}^{\alpha} \tau_{b}^{\beta} \tau_{c}^{\gamma} \tau_{d} \tau_{e}^{-2} \tau_{f}^{-2}$ is an open book on $S^{3}$ when

$$
(\alpha-1, \beta-3, \gamma-1)=(-1,-2,-4) .
$$

More generally, consider the braid $\beta=\left(\sigma_{2} \sigma_{1} \sigma_{2}\right)^{2} \sigma_{1}^{-2 p} \sigma_{2}^{-4}$ and perform Dehn surgery with coefficients $(1-p,-p,-4)$. In Figure 6 , we verify that we still obtain $S^{3}$.

By reflecting (which amounts to changing orientation), we also know that Dehn surgery on $\beta=\left(\sigma_{2} \sigma_{1} \sigma_{2}\right)^{-2} \sigma_{1}^{2 p} \sigma_{2}^{4}$ with coefficients $(p-1, p, 4)$ also yields $S^{3}$. 


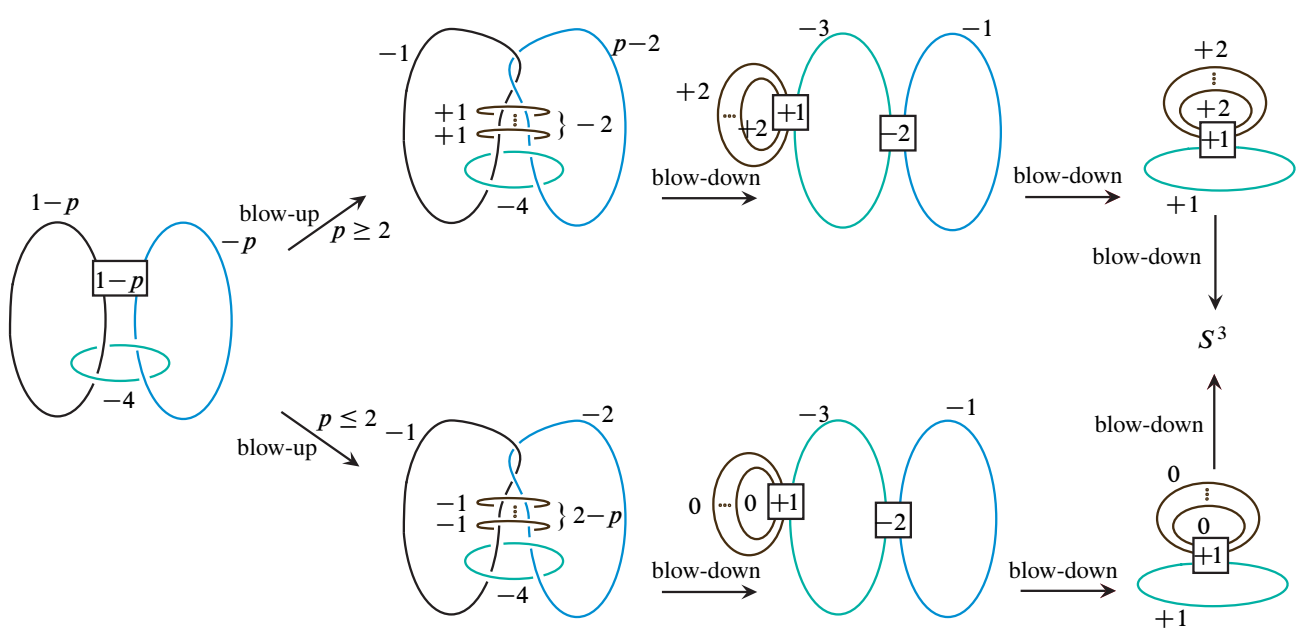

Figure 6: Surgery on a family of links yields $S^{3}$

After some experimentation, the author found that the following two families of open books (which are obtained from one another by reflecting the braid as above) will be sufficient for our purposes. (Note that reflecting the braid amounts to changing the orientation, but since $S^{3}$ has an orientation reversing diffeomorphism, this will still give an open book on $S^{3}$. Though, as we will see below the supported contact structure will change!)

It will suffice to consider the following two possibilities:

$$
\begin{aligned}
& \phi_{p}=\tau_{b} \tau_{c}^{-3} \tau_{d} \tau_{e}^{-p} \tau_{f}^{-2} \\
& \bar{\phi}_{p}=\tau_{b}^{-1} \tau_{c}^{3} \tau_{d}^{-1} \tau_{e}^{p} \tau_{f}^{2}
\end{aligned}
$$

We will denote the supported contact structures by $\xi_{p}$ and $\bar{\xi}_{p}$. Note that in both open books the monodromies have boundary parallel negative Dehn twists, it is easy to see that in this case, the monodromies are not right-veering. Therefore, the supported contact structures are overtwisted.

To determine the contact structures, following the description in [8] (see also [4]), we will next compute the $d_{3}$ invariants of the supported contact structures from the monodromy data of the open books. First, we briefly review the strategy, for more details see [8]. Given $\phi$ as a product of Dehn twists about homologically nontrivial curves $a_{1}, \ldots, a_{k}$ on a planar surface $S$ with $n$ boundary components, one first constructs the Stein manifold $S \times D^{2}$ in a standard way by attaching $n$ one-handles to $D^{4}$, then one attaches 2-handles along Legendrian realizations of $a_{i}$ on $S$ with \pm 1 framing 
depending on whether the Dehn twist about $a_{i}$ is left or right-handed. Let $W$ be the 4-manifold thus constructed. Then the contact manifold $(Y, \xi)$ supported by the open book $(S, \phi)$ is the boundary of $W$. As long as $c_{1}(\xi)=0$ (or more generally a torsion class) in $H^{2}(Y), d_{3}(\xi)$ is an element of $\mathbb{Q}$ and may be computed by the formula

$$
d_{3}(\xi)=\frac{1}{4}\left(c^{2}(W)-2 \chi(W)-3 \sigma(W)\right)+q
$$

where $q$ is the number of negative Dehn twists in the factorization of $\phi$. Furthermore, $c^{2}(W)$ is the square of the class $c(W) \in H^{2}(W)$ which is Poincare dual to the class $\sum_{i=1}^{k} \operatorname{rot}\left(a_{i}\right) C_{i} \in H_{2}(W, Y)$, where $C_{i}$ is the cocore of the 2-handle attached along $a_{i}$ and $\operatorname{rot}\left(a_{i}\right)$ is the rotation number of $a_{i}$ which can be computed as the winding number of $a_{i}$ with respect to a standard trivialization of the tangent bundle of the page. Since we assume $c_{1}(\xi)=0, c(W)$ maps to zero under the natural map $H^{2}(W) \rightarrow H^{2}(Y)$, hence it comes from class in $H^{2}(W, Y)$ whose square is $c^{2}(W)$ that appear in the formula above.

Now, Figure 7 is a Kirby diagram for the page of a planar open book with four boundary components. We drew all the curves $a, b, c, d, e$ and $f$ that appear in the above monodromies.

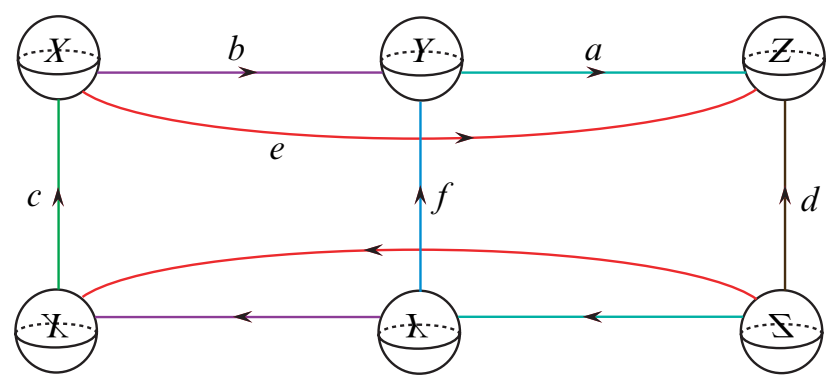

Figure 7: The diagram of the page

In order to compute the rotation numbers, we chose an orientation of the curves (note that the computation of $c^{2}(W)$ is independent of this choice). One then computes the winding numbers of these curves to get

$$
\begin{aligned}
& \operatorname{rot}(a)=\operatorname{rot}(b)=\operatorname{rot}(e)=1 \\
& \operatorname{rot}(c)=\operatorname{rot}(f)=\operatorname{rot}(d)=0 .
\end{aligned}
$$

The rest of the proof is a direct homology calculation based on the descriptions above. 
Computation of $d_{3}\left(\xi_{p}\right)$ and $d_{3}\left(\bar{\xi}_{p}\right)$ Let $X, Y$, and $Z$ be the 1 -handles, which form a basis of $C_{1}(W ; \mathbb{Z})$. Let $B,\left\{C_{1}, C_{2}, C_{3}\right\}, D,\left\{E_{1}, E_{2}, \ldots, E_{|p|}\right\},\left\{F_{1}, F_{2}\right\}$ be the cores of the handles attached corresponding to the factorization $\phi_{p}=\tau_{b} \tau_{c}^{-3} \tau_{d} \tau_{e}^{-p} \tau_{f}^{-2}$. These form a basis of $C_{2}(W ; \mathbb{Z})$ and the boundary map can be read off the diagram in Figure 7 to be

$$
\begin{aligned}
& d(B)=Y-X \quad d\left(C_{i}\right)=X \\
& d(D)=Z \quad d\left(D-E_{i}\right)=X \quad d\left(F_{i}\right)=Y .
\end{aligned}
$$

Thus, $H_{1}(W)=0$ and $H_{2}(W)=\mathbb{Z}^{|p|+4}$. It will be convenient to pick the following basis of generators:

$$
\begin{array}{r}
\left\{C_{1}-D+E_{|p|}, C_{1}-D+E_{|p|-1}, \ldots, C_{1}-D+E_{1}, B+C_{1}-F_{1}, B+C_{2}-F_{1}\right. \\
\left.B+C_{3}-F_{1}, F_{2}-F_{1}\right\}
\end{array}
$$

Note that we have $B^{2}=D^{2}=-1$ and $C_{1}^{2}=C_{2}^{2}=C_{3}^{2}=F_{1}^{2}=F_{2}^{2}=1$ and $E_{i}^{2}=$ $\operatorname{sgn}(p)$ and any cross term intersection number is zero. The intersection matrix takes particularly nice form if we add or subtract the first $p$ elements in the above basis to the $(p+1)-$ st element according to whether $p$ is negative or positive. So, our new basis is given by

$$
\begin{aligned}
& \left\{C_{1}-D+E_{|p|}, C_{1}-D+E_{|p|-1}, \ldots, C_{1}-D+E_{1},\right. \\
& B+(1-p) C_{1}+p D-F_{1}-\operatorname{sgn}(p) E_{1}-\cdots-\operatorname{sgn}(p) E_{|p|}, \\
& \left.B+C_{2}-F_{1}, B+C_{3}-F_{1}, F_{2}-F_{1}\right\}
\end{aligned}
$$

Therefore, the intersection matrix of $W$ in the this basis can be calculated to be:

$$
Q_{W}=\left(\begin{array}{ccccccc}
\operatorname{sgn}(p) & 0 & \ldots & \ldots & \ldots & \cdots & 0 \\
0 & \ddots & 0 & \ldots & \cdots & \cdots & 0 \\
\vdots & 0 & \operatorname{sgn}(p) & 0 & \cdots & \cdots & 0 \\
\vdots & \vdots & 0 & 1-p & 0 & 0 & 1 \\
\vdots & \vdots & \vdots & 0 & 1 & 0 & 1 \\
\vdots & \vdots & \vdots & 0 & 0 & 1 & 1 \\
0 & 0 & 0 & 1 & 1 & 1 & 2
\end{array}\right)
$$

From this one can easily compute that $\sigma(W)=2+p$, and also we know that $\chi(W)=$ $|p|+5$. To compute $c^{2}(W)$, let us denote the cocores by $\check{B},\left\{\check{C}_{1}, \check{C}_{2}, \breve{C}_{3}\right\}, \check{D}$, $\left\{\check{E}_{1}, \ldots, \check{E}_{p}\right\},\left\{\check{F}_{1}, \check{F}_{2}\right\}$. Then from the calculation of rotation numbers it follows that 
$c(W)$ is Poincaré dual to

$$
\check{B}+\check{E}_{1}+\ldots+\check{E}_{p}
$$

Evaluating $c(W)$ on our basis of $H_{2}(W)$, we get the vector $(1, \ldots, 1,1-p, 1,1,0)$ hence the Poincaré dual to the pull back of $c(W)$ to $H^{2}(W, Y)$ is given by $(1, \ldots, 1$, $1-p, 1,1,0)^{t} \cdot\left(Q_{W}\right)^{-1}$, which one can calculate to be:

$$
\left(\begin{array}{ccccccc}
\operatorname{sgn}(p) & 0 & \ldots & \cdots & \ldots & \ldots & 0 \\
0 & \ddots & 0 & \ldots & \ldots & \ldots & 0 \\
\vdots & 0 & \operatorname{sgn}(p) & 0 & \ldots & \ldots & 0 \\
\vdots & \vdots & 0 & 0 & -1 & -1 & 1 \\
\vdots & \vdots & \vdots & -1 & p & -1+p & 1-p \\
\vdots & \vdots & \vdots & -1 & -1+p & p & 1-p \\
0 & 0 & 0 & 1 & 1-p & 1-p & -1+p
\end{array}\right)\left(\begin{array}{c}
1 \\
\vdots \\
1 \\
1-p \\
1 \\
1 \\
0
\end{array}\right)
$$

Hence

$$
\begin{aligned}
c^{2}(W) & =(\operatorname{sgn}(p), \ldots, \operatorname{sgn}(p),-2,-2+3 p,-2+3 p, 3-3 p) \cdot(1, \ldots, 1,1-p, 1,1,0) \\
& =9 p-6
\end{aligned}
$$

The number of negative Dehn twists is given by $q(W)=5+(|p|+p) / 2$. Finally, we compute

$$
d_{3}\left(\xi_{p}\right)=\frac{1}{4}(9 p-6-2(|p|+5)-3(2+p))+5+\frac{|p|+p}{2}=2 p-\frac{1}{2} .
$$

This only covers half of the overtwisted contact structures on $S^{3}$, to get the other half, we consider $\bar{\xi}_{p}$. Note, that $\bar{\xi}_{p}$ is obtained by orientation reversal. Therefore, we do not need to compute all the above invariants from scratch. Namely, we have

$$
\begin{aligned}
c^{2}(-W) & =-c^{2}(W)=-9 p+6 \\
\chi(-W) & =\chi(W)=|p|+5 \\
\sigma(-W) & =-\sigma(W)=-p-2 \\
q(-W) & =2+\frac{|p|-p}{2} .
\end{aligned}
$$

Therefore, we have

$$
d_{3}\left(\bar{\xi}_{p}\right)=\frac{1}{4}(-9 p+6-2(|p|+5)-3(-p-2))+2+\frac{|p|-p}{2}=-2 p+\frac{5}{2} .
$$

We determined the binding number of all the overtwisted contact structures. The proof of Theorem 1.1 will be completed once we determine the support norm of 
the overtwisted contact structures. Note that because all of the overtwisted contact structures are supported by a planar open book with page a four-holed sphere, we have that $\operatorname{sn}(\xi) \leq 2$ for all $\xi$. We also know that if $d_{3}(\xi) \neq-1 / 2,1 / 2,3 / 2$, then $\operatorname{bn}(\xi)=4$, therefore the only way for these contact structures to have support norm strictly less than 2 is when they are supported by an open book with page a torus with one boundary component. Now, recall the well-known fact that the only genus one fibred knots on $S^{3}$ are trefoil and figure-eight knot and the corresponding open books have monodromy $\tau_{a}^{ \pm 1} \tau_{b}^{ \pm 1}$, where $a$ and $b$ are standard generators of the homology of the torus, (this follows from for example [17]). It is now easy to see that $\tau_{a} \tau_{b}, \tau_{a} \tau_{b}^{-1}, \tau_{a}^{-1} \tau_{b}$ are obtained by positively stabilizing the open books with annulus page supporting the unique tight contact structure, and the overtwisted contact structure $\xi_{1 / 2}$, and $\tau_{a}^{-1} \tau_{b}^{-1}$ is obtained by negatively stabilizing $\xi_{1 / 2}$, hence corresponds to $\xi_{3 / 2}$. This completes the proof of Theorem 1.1.

\begin{tabular}{|c|c|c|c|c|c|}
\cline { 2 - 6 } \multicolumn{1}{c|}{} & $n<-1 / 2$ & $n=-1 / 2$ & $n=1 / 2$ & $n=3 / 2$ & $n>3 / 2$ \\
\hline bn & 4 & 3 & 2 & 3 & 4 \\
\hline sn & 2 & 1 & 0 & 1 & 2 \\
\hline sg & 0 & 0 & 0 & 0 & 0 \\
\hline
\end{tabular}

\begin{tabular}{|c|}
\hline$\xi_{\mathrm{st}}$ \\
\hline 1 \\
\hline-1 \\
\hline 0 \\
\hline
\end{tabular}

\section{Positive factorizations}

In this section we give a proof of Theorem 1.3 in the following two propositions. Recall that for the four-holed sphere $S, \operatorname{Map}(S, \partial S)=\mathbb{Z}^{4} \times F_{2}$. The first homology is

$$
H_{1}(\operatorname{Map}(S, \partial S))=\mathbb{Z}^{6}
$$

where the class of a general element $\phi=\tau_{a}^{\alpha} \tau_{b}^{\beta} \tau_{c}^{\gamma} \tau_{d}^{\delta} \tau_{e}^{\epsilon_{1}} \tau_{f}^{\eta_{1}} \cdots \tau_{e}^{\epsilon_{k}} \tau_{f}^{\eta_{k}}$ is given by $\left(\alpha, \beta, \gamma, \delta, \sum_{i=1}^{k} \epsilon_{k}, \sum_{i=1}^{k} \eta_{k}\right)$. We will prove the following proposition:

Proposition 3.1 If $\phi=\tau_{a}^{\alpha} \tau_{b}^{\beta} \tau_{c}^{\gamma} \tau_{d}^{\delta} \tau_{e}^{\epsilon} \tau_{f}^{\eta}$, then $\phi$ admits a positive factorization if and only if $\min \{\alpha, \beta, \gamma, \delta\} \geq \max \{-\epsilon,-\eta, 0\}$.

Proof Suppose $\phi=\tau_{C_{1}} \cdots \tau_{C_{k}}$ is a positive factorization in $\operatorname{Map}(S, \partial S)$. We consider the quotient relation in $H_{1}(\operatorname{Map}(S, \partial S))=\mathbb{Z}^{6}$ :

$$
(\alpha, \beta, \gamma, \delta, \epsilon, \eta)=\left[\tau_{C_{1}}\right]+\cdots+\left[\tau_{C_{k}}\right]
$$


Now, by topological classification of surfaces observe that any simple closed curve $C_{i}$ is conjugate in $\operatorname{Map}(S, \partial S)$ to one of the curves $a, b, c, d, e, f$ or $g$ in Figure 2. The classes of Dehn twists around these curves in $H_{1}(\operatorname{Map}(S, \partial S))$ are given by $\left[\tau_{a}\right]=$ $(1,0,0,0,0,0),\left[\tau_{b}\right]=(0,1,0,0,0,0),\left[\tau_{c}\right]=(0,0,1,0,0,0),\left[\tau_{d}\right]=(0,0,0,1,0,0)$, $\tau_{e}=(0,0,0,0,1,0),\left[\tau_{f}\right]=(0,0,0,0,0,1)$ and $\left[\tau_{g}\right]=(1,1,1,1,-1,-1)$. For the latter, observe that by the lantern relation we have $\tau_{g}=\tau_{a} \tau_{b} \tau_{c} \tau_{d} \tau_{e}^{-1} \tau_{f}^{-1}$. Let us denote by $e_{i} \in \mathbb{Z}^{6}$ the vector with $i$-th coordinate 1 and other coordinates 0 and let $n=(1,1,1,1,-1,-1)$. Therefore, each class $\left[\tau_{C_{i}}\right]$ is equal to either some $e_{j}$ or $n$. Now, if $\phi$ has positive factorization then

$$
(\alpha, \beta, \gamma, \delta, \epsilon, \eta)=p_{0} n+\sum_{i=1}^{6} p_{i} e_{i}
$$

for some $p_{i} \geq 0$. Thus if $\epsilon$ or $\eta$ is negative, $p_{0} \geq \max \{-\epsilon,-\eta\}$, which shows that $\min \{\alpha, \beta, \gamma, \delta\} \geq \max \{-\epsilon,-\eta, 0\}$ as desired.

Conversely, if $\epsilon, \eta \geq 0$, then the given factorization is positive if $\min \{\alpha, \beta, \gamma, \delta\}>0$. Without loss of generality, suppose next that $\epsilon<0$ and $\eta=\epsilon+r$ for $r \geq 0$. We have $\min \{\alpha, \beta, \gamma, \delta\} \geq-\epsilon$, set $-\epsilon=k>0$. Then by using the lantern relation $k$ times, we obtain the central element $\left(\tau_{f} \tau_{e} \tau_{g}\right)^{k}$. We first use this to kill the negative powers of $f$, to get

$$
\begin{aligned}
\phi & =\tau_{a}^{\alpha-k} \tau_{b}^{\beta-k} \tau_{c}^{\gamma-k} \tau_{d}^{\delta-k} \tau_{e}^{-k} \tau_{f}^{-k}\left(\tau_{f} \tau_{e} \tau_{g}\right)^{k} \tau_{f}^{r} \\
& =\tau_{a}^{\alpha-k} \tau_{b}^{\beta-k} \tau_{c}^{\gamma-k} \tau_{d}^{\delta-k} \tau_{e}^{-k} \tau_{f}^{-k} \tau_{f}^{k}\left(\tau_{e} \tau_{g}\right)^{k} \tau_{f}^{r} \\
& =\tau_{a}^{\alpha-k} \tau_{b}^{\beta-k} \tau_{c}^{\gamma-k} \tau_{d}^{\delta-k} \tau_{e}^{-k+1} \tau_{g}\left(\tau_{e} \tau_{g}\right)^{k-1} \tau_{f}^{r} .
\end{aligned}
$$

The proof will be completed once we show that $\tau_{e}^{-k+1} \tau_{g}\left(\tau_{e} \tau_{g}\right)^{k-1}$ has a positive factorization. We do this by induction and using the well-known fact that if $f: S \rightarrow S$ a diffeomorphism and $C$ a simple closed curve then the equality $\tau_{f(C)}=f^{-1} \tau_{C} f$ holds. For $k=1$, the expression is equal to $\tau_{g}$ so it is positive. We write

$$
\begin{aligned}
\tau_{e}^{-k+1} \tau_{g}\left(\tau_{e} \tau_{g}\right)^{k-1} & =\tau_{e}^{-k+1} \tau_{g} \tau_{e}^{-(-k+1)} \tau_{e}^{-k+2} \tau_{g}\left(\tau_{e} \tau_{g}\right)^{k-2} \\
& =\tau_{\tau_{e}^{k-1}(g)} \tau_{e}^{-k+2} \tau_{g}\left(\tau_{e} \tau_{g}\right)^{k-2} .
\end{aligned}
$$

The latter expression is positive by induction hypothesis, which completes the proof. In fact, we can simply see that

$$
\phi=\tau_{a}^{\alpha-k} \tau_{b}^{\beta-k} \tau_{c}^{\gamma-k} \tau_{d}^{\delta-k} \tau_{\tau_{e}^{k-1}(g)} \tau_{\tau_{e}^{k-2}(g)} \cdots \tau_{\tau_{e}(g)} \tau_{g} \tau_{f}^{r} .
$$

Remark 3.2 Suppose more generally that $\phi=\tau_{a}^{\alpha} \tau_{b}^{\beta} \tau_{c}^{\gamma} \tau_{d}^{\delta} \tau_{e}^{\epsilon_{1}} \tau_{f}^{\eta_{1}} \cdots \tau_{e}^{\epsilon_{k}} \tau_{f}^{\eta_{k}}$. Let $\epsilon=\sum_{i=1}^{k} \epsilon_{k}$ and $\eta=\sum_{i=1}^{k} \eta_{k}$. Then the same argument using $H_{1}(\operatorname{Map}(S, \partial S))$ 
gives that $\phi$ has a positive factorization only if $\min \{\alpha, \beta, \gamma, \delta\} \geq \max \{-\epsilon,-\eta, 0\}$. However, it is easy to see that this is not sufficient. For example, $\tau_{e} \tau_{f} \tau_{e}^{-1} \tau_{f}^{-1}$ satisfies this condition, but one can check that this is not a right-veering monodromy hence cannot be written as a product of right-handed Dehn twists (by [15] the supported contact structure can not even be tight). On the other hand, the argument given in the above proof clearly gives a positive factorization of $\phi$ if $\min \{\alpha, \beta, \gamma, \delta\} \geq \sum_{i=1}^{k} \max \left\{\epsilon_{i}, \eta_{i}, 0\right\}$.

We next determine whether or not the Ozsváth-Szabó contact invariant vanishes for these contact structures. In our case, it turns out that this is equivalent to whether or not the contact structure is Stein fillable.

Proposition 3.3 If $\phi=\tau_{a}^{\alpha} \tau_{b}^{\beta} \tau_{c}^{\gamma} \tau_{d}^{\delta} \tau_{e}^{\epsilon} \tau_{f}^{\eta}$, then the contact invariant $c^{+}(\phi)$ is nonzero if and only if $\min \{\alpha, \beta, \gamma, \delta\} \geq \max \{-\epsilon,-\eta, 0\}$.

Proof Let us define $\phi_{a}, \phi_{b}, \phi_{c}, \phi_{d}$ to be the induced monodromies on the three-holed planar surface after one "caps off" the boundary components parallel to $a, b, c$ or $d$ by gluing a disk to the corresponding boundary component and extending the monodromy by identity on this disk. Let $\xi_{a}, \xi_{b}, \xi_{c}$ and $\xi_{d}$ be the corresponding contact structures obtained this way.

In [3, Corollary 1.3], Baldwin proves that if the contact invariant of any of the contact structures $\xi_{a}, \xi_{b}, \xi_{c}$ and $\xi_{d}$ is zero, then it must be the case that $c^{+}(\phi)=0$. Without loss of generality, suppose that $\epsilon=-k<0$, and $\alpha<k$. Then let's consider the open book $\phi_{b}$ where the boundary component parallel to the curve $b$ is capped off. In that case, $e$ becomes isotopic to $a$, and no other curves become isotopic to these pair. Therefore, the monodromy $\phi_{b}$ has $k-\alpha$ left-handed Dehn twists around the boundary component corresponding to $a$, which shows that the supported contact structure $\xi_{b}$ is overtwisted [15]. Therefore, the contact invariant $c^{+}\left(\xi_{b}\right)=0$ by [21]. Hence, it follows that $c^{+}(\phi)=0$.

Conversely, if $\min \{\alpha, \beta, \gamma, \delta\} \geq \max \{-\epsilon,-\eta, 0\}$, then by the previous proposition, the supported contact structure is Stein fillable, hence $c^{+}(\phi) \neq 0$ by [21].

Remark 3.4 As in the previous remark, for the more general class of diffeomorphisms, $\phi=\tau_{a}^{\alpha} \tau_{b}^{\beta} \tau_{c}^{\gamma} \tau_{d}^{\delta} \tau_{e}^{\epsilon_{1}} \tau_{f}^{\eta_{1}} \cdots \tau_{e}^{\epsilon_{k}} \tau_{f}^{\eta_{k}}$, the above argument shows that $c^{+}(\phi)=0$ when $\min \{\alpha, \beta, \gamma, \delta\}<\max \{-\epsilon,-\eta, 0\}$. However, the converse is not true again by the same example given there, namely $\tau_{e} \tau_{f} \tau_{e}^{-1} \tau_{f}^{-1}$ has vanishing $c^{+}(\phi)=0$, since it supports an overtwisted contact structure [21]. We would like to point out that this is an overtwisted contact structure on $T^{3}$. 


\section{An example}

\subsection{Poincaré homology sphere}

Once we have the surgery description on pure 3-braid closures, it is easy to play around with simple 3-braids and surgeries on them to get interesting open book decompositions on various manifolds.

Proof of Theorem 1.2 The simplest nontrivial pure 3-braid is arguably the braid $\beta=\left(\sigma_{2} \sigma_{1} \sigma_{2}\right)^{2} \sigma_{1}^{-4}$ whose braid closure is shown in Figure 8 .

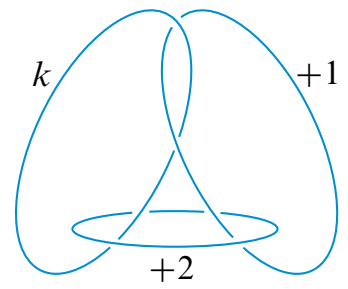

Figure 8: The braid closure of $\beta=\left(\sigma_{2} \sigma_{1} \sigma_{2}\right)^{2} \sigma_{1}^{-4}$

We first verify below that if we do surgery on the closure of this braid with surgery coefficients $(k, 1,2)$, then the result is $k-5$ surgery on the left-handed trefoil. By Proposition 2.2, we obtain an open book with page a four-holed sphere and $\phi=$ $\tau_{a}^{k+1} \tau_{b}^{2} \tau_{c} \tau_{d} \tau_{e}^{-2}$. In particular, the $(4,1,2)$ surgery yields the infamous Poincaré homology sphere which has a unique tight contact structure.

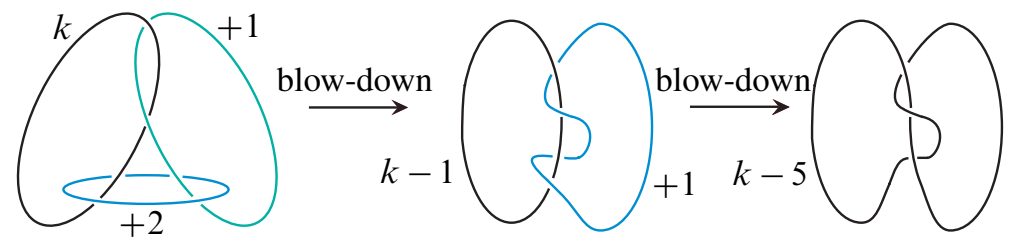

Figure 9: Surgery on the left-handed trefoil

It is easy to see that the monodromy $\phi=\tau_{a}^{k+1} \tau_{b}^{2} \tau_{c} \tau_{d} \tau_{e}^{-2}$ is right-veering. In fact, the monodromy is given by a multicurve, and it is easy to see that all the boundary components are protected. To be more careful, since the right-veering diffeomorphisms is a submonoid of $\operatorname{Aut}(S, \partial S)$, it suffices to show that $\tau_{a} \tau_{e}^{-2}$ is right-veering with respect to the boundary component encircled by $a$. To see this, we can apply the Lemma 3.3 of [15]. We first put a hyperbolic structure on $S$ so that the boundary 
components are geodesics. Next, we need to find a subsurface $S^{\prime}$ of $S$ with geodesic boundary so that the boundary component of $S$ encircled by $a$ is a common boundary component of $S$ and $S^{\prime}$ and that $\tau_{e}^{-2}$ is identity on $S^{\prime}$. This is easily accomplished by taking $S^{\prime}$ to be the pair-of-pants that has boundary at the boundary components encircled by $a$ and $b$ and also a geodesic curve which encircles both $a$ and $b$ (parallel to $e$ ). Note that by isotoping $e$ if necessary, we can arrange that $S^{\prime}$ is disjoint from $e$ and has geodesic boundary. Thus Lemma 3.3 of [15] applied as above to each boundary component of $S$ shows that $\phi=\tau_{a}^{k+1} \tau_{b}^{2} \tau_{c} \tau_{d} \tau_{e}^{-2}$ is right-veering for $k \geq 0$.

We next prove that $(S, \phi)$ is also not destabilizable. If it were, then it would be a stabilization of an open book $\left(P, \phi^{\prime}\right)$, with page $P$ a three-holed sphere, where $\phi^{\prime}=\tau_{a}^{p} \tau_{b}^{q} \tau_{c}^{r}$ for the curves $a, b$ and $c$ are as in Figure 1 . As we noted before, such a $\left(P, \phi^{\prime}\right)$ is an open book on a Seifert fibred space with $e_{0}=\lfloor-1 / p\rfloor+\lfloor-1 / q\rfloor+\lfloor-1 / r\rfloor$. Now, since $e_{0}$ for the Poincare homology sphere is -2 , it follows that at least one of the exponents $p, q$ or $r$ is negative (for example, $\tau_{a}^{-2} \tau_{b}^{3} \tau_{c}^{5}$ is an open book on the Poincaré homology sphere). Any stabilization which gives a page with four holed sphere must be obtained by attaching a 1 -handle $h$ to a fixed boundary component of $P$ and introducing a new monodromy curve $s$ which intersects the cocore of $h$ at a unique point, so that $\phi=\tau_{s} \phi^{\prime}$, where $\phi^{\prime}$ is extended by identity along $h$ to a diffeomorphism of $S$. We now argue that the monodromy of every such open book is not right-veering. Indeed, by noting that the curve $s$ is constraint to intersect the cocore of $h$ (which is a properly embedded arc that connects the top two boundary components of $S$, in Figure 10), it is easy to see that one could always find a diffeomorphism of $S$ (not necessarily fixing boundary components but sending boundary components to boundary components), such that the configuration of monodromy curves in $\tau_{s} \phi^{\prime}$ is as in Figure 10, where the sets of curves $x, y$ and $z$ depicted are a permutation of the images of the sets of curves corresponding to Dehn twists around $a, b$ and $c$ after stabilization. Indeed, $S$ is composed of two pairs-of-pants, separated by a curve parallel to $x$ curves in Figure 10, and noting the fact that $s$ intersects cocore of $h$ at a unique point, we can arrange by isotopy so that either it does not intersect the common boundary of the two pair-of-pants or it intersects at precisely two points, and now apply a diffeomorphism of the pair-of-pants at the bottom, which fixes the boundary component parallel to $x$ curves, but rotates the other boundary components if necessary. Finally, since we know that at least one of the sets of curves $x, y$ or $z$ are all negative Dehn twists, by looking at Figure 10, it is now easy to see that the monodromy $\tau_{s} \phi^{\prime}$ is not right-veering. This proves that $\phi$ cannot be destabilized.

On the other hand, it follows from the obstruction result of [6] that the unique tight contact structure on the Poincare homology sphere cannot support a planar open book. (Alternatively, it is known that the unique tight contact structure on the Poincaré 

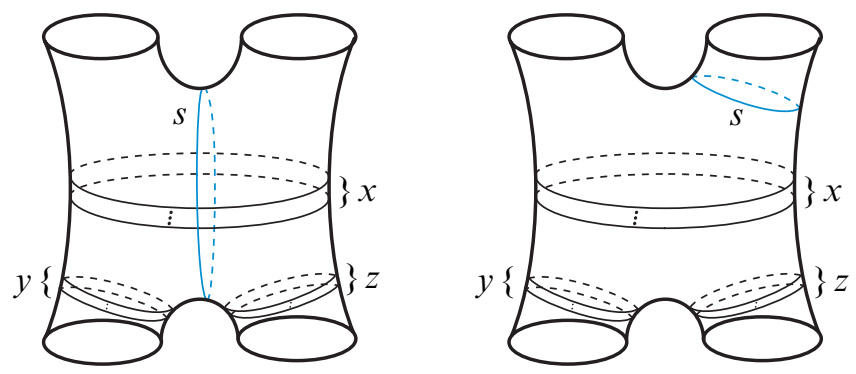

Figure 10: Stabilized open books

homology sphere is Stein fillable, hence, by the results of Wendl [25], if a planar open book supports this contact structure, it should have a positive factorization but this contradicts our Theorem 1.3. )

Therefore, the contact structure supported by the open book $\left(S, \tau_{a}^{5} \tau_{b}^{2} \tau_{c} \tau_{d} \tau_{e}^{-2}\right)$ is an overtwisted one, which completes the proof of our Theorem 1.2.

Remark 4.1 Note that the way we argued for the overtwistedness of $\tau_{a}^{5} \tau_{b}^{2} \tau_{c} \tau_{d} \tau_{e}^{-2}$ is quite special to the case of Poincaré homology sphere. In particular, we used the fact that the unique tight contact structure on this manifold is not supported by a planar open book. A similar argument can be made for $k=0,1,2,3$ to obtain rightveering, not destabilizable monodromies which support overtwisted contact structures (using the classification result in [11], which in particular says that all the tight contact structures on these manifolds are Stein fillable). However, we do not know if $\tau_{a}^{k+1} \tau_{b}^{2} \tau_{c} \tau_{d} \tau_{e}^{-2}$ is overtwisted or tight for $k>4$. These are Seifert fibered manifolds $M(-2 ; 1 / 2,2 / 3, k /(k+1))$, they have $e_{0}(M)=-2$ but they are not L-spaces. The classification of tight contact structures on these Seifert manifolds seems not yet to have been completed. Note that the corresponding monodromies are all right-veering diffeomorphisms and the contact invariants of the corresponding contact structures are zero.

\section{References}

[1] C Adams, W Sherman, Minimum ideal triangulations of hyperbolic 3-manifolds, Discrete Comput. Geom. 6 (1991) 135-153 MR1083629

[2] L Armas-Sanabria, M Eudave-Muñoz, The hexatangle, Topology Appl. 156 (2009) 1037-1053 MR2493367

[3] J Baldwin, Capping off open books and the Ozsváth-Szabó contact invariant arXiv: 0901.3797 
[4] J Baldwin, J B Etnyre, A note on the support norm of a contact structure, to appear in "Proceedings of the 2009 Georgia Topology Conference (Athens, GA)" arXiv: 0910.5021

[5] T Etgü, Y Lekili, Examples of planar tight contact structures with support norm one, Int. Math. Res. Not. (2010) 3723-3728 MR2725510

[6] J B Etnyre, Planar open book decompositions and contact structures, Int. Math. Res. Not. (2004) 4255-4267 MR2126827

[7] J B Etnyre, Lectures on open book decompositions and contact structures, from: "Floer homology, gauge theory, and low-dimensional topology", (D A Ellwood, P S Ozsváth, A I Stipsicz, Z Szabó, editors), Clay Math. Proc. 5, Amer. Math. Soc. (2006) 103-141 MR2249250

[8] J B Etnyre, B Ozbagci, Invariants of contact structures from open books, Trans. Amer. Math. Soc. 360 (2008) 3133-3151 MR2379791

[9] E Fadell, L Neuwirth, Configuration spaces, Math. Scand. 10 (1962) 111-118 MR0141126

[10] R Fox, L Neuwirth, The braid groups, Math. Scand. 10 (1962) 119-126 MR0150755

[11] P Ghiggini, On tight contact structures with negative maximal twisting number on small Seifert manifolds, Algebr. Geom. Topol. 8 (2008) 381-396 MR2443233

[12] E Giroux, Géométrie de contact: de la dimension trois vers les dimensions supérieures, from: "Proceedings of the International Congress of Mathematicians, Vol. II (Beijing, 2002)", (T Li, editor), Higher Ed. Press, Beijing (2002) 405-414 MR1957051

[13] C M Gordon, Small surfaces and Dehn filling, from: "Proceedings of the Kirbyfest (Berkeley, CA, 1998)", (J Hass, M Scharlemann, editors), Geom. Topol. Monogr. 2, Geom. Topol. Publ., Coventry (1999) 177-199 MR1734408

[14] C M Gordon, Y-Q Wu, Toroidal and annular Dehn fillings, Proc. London Math. Soc. (3) 78 (1999) 662-700 MR1674841

[15] K Honda, W H Kazez, G Matić, Right-veering diffeomorphisms of compact surfaces with boundary, Invent. Math. 169 (2007) 427-449 MR2318562

[16] K Honda, W H Kazez, G Matić, On the contact class in Heegaard Floer homology, J. Differential Geom. 83 (2009) 289-311 MR2577470

[17] W Magnus, A Peluso, On knot groups, Comm. Pure Appl. Math. 20 (1967) 749-770 MR0222880

[18] B Martelli, C Petronio, Dehn filling of the "magic" 3-manifold, Comm. Anal. Geom. 14 (2006) 969-1026 MR2287152

[19] K Niederkrüger, C Wendl, Weak symplectic fillings and holomorphic curves, to appear in Ann. Sci. École Norm. Sup. arXiv:1003.3923 
[20] P Ozsváth, A Stipsicz, Z Szabó, Planar open books and Floer homology, Int. Math. Res. Not. (2005) 3385-3401 MR2200085

[21] P Ozsváth, Z Szabó, Heegaard Floer homology and contact structures, Duke Math. J. 129 (2005) 39-61 MR2153455

[22] V V Prasolov, A B Sossinsky, Knots, links, braids and 3-manifolds. An introduction to the new invariants in low-dimensional topology, Translations of Math. Monogr. 154, Amer. Math. Soc. (1997) MR1414898 Translated from the Russian manuscript by Sossinsky

[23] W P Thurston, The geometry and topology of three-manifolds, Princeton Univ. Math. Dept. Lecture Notes (1979) Available at http://msri.org/publications/books/ gt $3 \mathrm{~m} /$

[24] A Wand, Mapping class group relations, Stein fillings, and planar open book decompositions arXiv:1006.2550

[25] C Wendl, Strongly fillable contact manifolds and J-holomorphic foliations, Duke Math. J. 151 (2010) 337-384 MR2605865

Department of Pure Mathematics and Mathematical Statistics, University of Cambridge Wilberforce Road, Cambridge, CB3 OWA, UK

y1319@cam.ac.uk

http://people.pwf.cam.ac.uk/yl319/

Received: 17 September 2010 